MITSUBISHI ELECTRIC RESEARCH LABORATORIES

http://www.merl.com

\title{
An Iterative Approach to the Optimal Co-Design of Linear Control Systems
}

\author{
Jiang, Y.; Wang, Y.; Bortoff, S.A.; Jiang, Z.-P. \\ TR2015-117 October 2015
}

\begin{abstract}
This paper investigates the optimal co-design of both physical plants and control policies for a class of continuous time linear control systems. The optimal co-design of a specific linear control system is commonly formulated as a nonlinear non-convex optimization problem (NNOP), and solved by using iterative techniques, where the plant parameters and the control policy are updated iteratively and alternately. This paper proposes a novel iterative approach to solve the NNOP, where the plant parameters are updated by solving a standard semi-definite programming problem, with non-convexity no longer involved. The proposed system design is generally less conservative in terms of the system performance compared to the conventional system-equivalence-based design, albeit the range of applicability is slightly reduced. A practical optimization algorithm is proposed to compute a sub-optimal solution ensuring the system stability, and the convergence of the algorithm is established. The effectiveness of the proposed algorithm is illustrated by its application to the optimal co-design of a physical load positioning system.
\end{abstract}

2015 International Journal of Control

\begin{abstract}
This work may not be copied or reproduced in whole or in part for any commercial purpose. Permission to copy in whole or in part without payment of fee is granted for nonprofit educational and research purposes provided that all such whole or partial copies include the following: a notice that such copying is by permission of Mitsubishi Electric Research Laboratories, Inc.; an acknowledgment of the authors and individual contributions to the work; and all applicable portions of the copyright notice. Copying, reproduction, or republishing for any other purpose shall require a license with payment of fee to Mitsubishi Electric Research Laboratories, Inc. All rights reserved.
\end{abstract}





\title{
An Iterative Approach to the Optimal Co-Design of Linear Control Systems
}

\author{
Yu Jiang ${ }^{\mathrm{a}}$, Yebin Wang ${ }^{\mathrm{b} *}$, Scott A. Bortoff ${ }^{\mathrm{b}}$ and Zhong-Ping Jiang ${ }^{\mathrm{c}}$ \\ ${ }^{a}$ The MathWorks, Inc., 3 Apple Hill Dr, Natick, MA 01760, USA. \\ ${ }^{b}$ Mitsubishi Electric Research Laboratories, 201 Broadway, Cambridge, MA 02139, USA. \\ ${ }^{c}$ Department of Electrical and Computer Engineering, Polytechnic School of Engineering, New York University, \\ Brooklyn, NY 11201, USA. \\ ( 4.0 released September 2014$)$
}

Keywords: Co-design; optimal control; non-convex optimization; iterative scheme.

\begin{abstract}
This paper investigates the optimal co-design of both physical plants and control policies for a class of continuoustime linear control systems. The optimal co-design of a specific linear control system is commonly formulated as a nonlinear non-convex optimization problem (NNOP), and solved by using iterative techniques, where the plant parameters and the control policy are updated iteratively and alternately. This paper proposes a novel iterative approach to solve the NNOP, where the plant parameters are updated by solving a standard semi-definite programming problem, with non-convexity no longer involved. The proposed system design is generally less conservative in terms of the system performance compared to the conventional system-equivalence-based design, albeit the range of applicability is slightly reduced. A practical optimization algorithm is proposed to compute a sub-optimal solution ensuring the system stability, and the convergence of the algorithm is established. The effectiveness of the proposed algorithm is illustrated by its application to the optimal co-design of a physical load positioning system.
\end{abstract}

\section{Introduction}

Optimal control theory tries to solve control policy problems for a given dynamic system such that certain optimality objectives can be achieved (Bertsekas, 1995; Bryson and Ho, 1975; Lewis et al., 2012). From a conventional perspective of control policy design, the plant is given at the control design stage, and thus the determination of the control policy is separate from the plant design. This separation may simplify the design processes of both the plant and the control policy but sacrifice the potential performance improvement brought by co-design, i.e., simultaneous design of the plant and the control policy. The performance loss due to the decoupled plant and control design is understood by reviewing numerous results on the performance limitation analysis of linear control systems, e.g. Bode (1945); Freudenberg and Looze (1985); Serön et al. (1997). Indeed, co-design problems can find a great number of applications, such as the optimal design and control of aerospace crafts (Hale et al., 1985; Messac, 1998), smart buildings (Lu and Skelton, 2000; Skelton and Kim, 1992), electromechanical devices (da Silva et al., 2009; Peters et al., 2011; Reyer and Papalambros, 2002), and robotics (Ravichandran et al., 2006).

The study of co-design problems in mechanical and aeronautics communities can be traced back to the 1980s, and leads to several strategies including iterative, bi-level (nested), and simultaneous (Fathy et al., 2001). An earlier approach is to numerically and simultaneously optimize the parameters of both the plant and the control policy using nonlinear programming (NLP) strategies (Onoda and Haftka, 1987; Salama

*Corresponding author. Email: yebinwang@ieee.org 
et al., 1988). Necessary optimality conditions and the coupling of co-design problems have been studied (Alyaqout et al., 2007; Fathy et al., 2001; Patil et al., 2010, 2012; Peters et al., 2010, 2011). In the aforementioned prior art, the non-convex co-design problem is tackled by direct transcript to NLP solvers, which suffer some well-known weaknesses, for instance sensitivity to initial guesses, no convergence guarantee etc. Also, turning to generic NLP solvers may compromise the efficiency, due to their lack of mechanisms to fully exploit the structure of co-design problems. Another class of schemes is based on iterative designs (Grigoriadis et al., 1993; Li et al., 2001; Lu and Skelton, 2000; Pil and Asada, 1996; Skelton and Kim, 1992). To guarantee the stability and the convergence properties, a key system equivalence constraint is imposed (Grigoriadis et al., 1993; Jiang et al., 2015; Lu and Skelton, 2000; Skelton and Kim, 1992), such that updating the system parameters will not compromise the closed-loop system performance. The inclusion of the system equivalence constraint is however not necessary and may restrict the feasible set of the co-design problem.

The primary objective of this paper is to develop a novel and systematic iterative technique for solving systematically co-design problems for a class of continuous-time linear control systems. Given some initial system parameters and an initial stabilizing control policy, we develop methods to improve the system parameters and the control policy sequentially in each iteration step without compromising the closed-loop system performance. The proposed methodology eliminates the non-convex system equivalence constraint, thus may weaken the restriction on the feasible set. We further show that for a plant with linear parameterizations, the system parameters can be improved by solving a standard semi-definite programming (SDP) problem (Boyd and Vandenberghe, 2004). The proposed iterative technique is systematic because of the explicit process of reducing the system parameter design to an SDP problem, and the fact that the global optimum of the resultant SDP problem can be reliably computed by numerous solvers. The efficiency of the proposed iterative method is illustrated through the application to the optimal co-design problem of a load positioning system (Shilpiekandula et al., 2012). It is worth pointing out that the iterative idea has been widely adopted in existing work including Grigoriadis et al. (1993); Lu and Skelton (2000); Skelton and Kim (1992), and the iterative process is essentially the same as the bi-level (nested) approach in (Fathy et al., 2001), which focus on co-design strategies for nonlinear systems instead of algorithms.

The remainder of this paper is organized as follows. Section 2 gives the problem formulation and motivations. Section 3 develops the novel iterative technique for solving optimal co-design problems for continuous-time linear control systems. A convergence proof is given. Section 4 illustrates the proposed approach on a design example of the load positioning problem. Finally, conclusions are given in Section 5.

\section{Problem formulation and preliminaries}

Consider the linear time-invariant control system

$$
\begin{aligned}
& \dot{x}=A(\theta) x+B(\theta) u \\
& y=C(\theta) x+D(\theta) u
\end{aligned}
$$

where $x \in \mathrm{R}^{n}$ is the system state, $u \in \mathrm{R}^{m}$ is the control input, and $\theta \in \mathrm{R}^{l}$ is the vector of system parameters to be designed. The system matrices $A(\theta) \in \mathrm{R}^{n \times n}$ and $B(\theta) \in \mathrm{R}^{n \times m}$ are linear in $\theta$, i.e., $\theta=\left[\boldsymbol{\theta}^{(1)}, \boldsymbol{\theta}^{(2)}, \cdots, \boldsymbol{\theta}^{(l)}\right]^{T}, A(\theta)=\sum_{j=1}^{l} \boldsymbol{\theta}^{(j)} A^{(j)}$, and $B(\theta)=\sum_{j=1}^{l} \theta^{(j)} B^{(j)}$. For simplicity, this paper considers full state feedback control, i.e., $C(\theta)=I_{n}$ and $D(\theta)=0$. The system parameter vector $\theta$ has $\theta_{\max } \in \mathrm{R}^{l}$ and $\theta_{\min } \in \mathrm{R}^{l}$ as its component-wise upper and lower bounds, i.e., the $i$ th component of $\theta$ is lower and upper bounded by the ith component of $\theta_{\min }$ and $\theta_{\max }$, respectively. For simplicity of notation, we denote the constraint on $\theta$ as $\theta_{\min } \leq \theta \leq \theta_{\max }$. The pair $[A(\theta), B(\theta)]$ is assumed to be stabilizable, for any $\theta$ satisfying $\theta_{\min } \leq \theta \leq \theta_{\max }$

Generally, a co-design problem may have multiple competing objectives capturing various design specifications, e.g. economical/environmental costs, control system performance, and maintainability. This paper considers a special co-design problem where the co-design objective is to find simultaneously a vector $\theta$ 
and a linear control policy $u=K x$ such that the following control objective is minimized

$$
J\left(x_{0}, \theta, K\right)=\int_{0}^{\infty}\left(x^{T} Q x+u^{T} R u\right) d t, x(0)=x_{0}
$$

where $Q=Q^{T} \geq 0, R=R^{T}>0$, and the pair $\left[A(\theta), Q^{1 / 2}\right]$ is assumed to be observable, if $\theta_{\min } \leq \theta \leq \theta_{\max }$.

To guarantee the existence of a finite cost with non-trivial initial state, it is natural to require that the closed-loop system matrix

$$
A_{c}(\theta, K):=A(\theta)+B(\theta) K
$$

is Hurwitz. Then, by linear optimal control theory, see e.g. Lewis et al. (2012), there exists a symmetric and positive definite matrix $P$ (called cost matrix), such that

$$
\begin{aligned}
J\left(x_{0}, \theta, K\right) & =x_{0}^{T} \int_{0}^{\infty} e^{A_{c}(\theta, K)^{T} t}\left(Q+K^{T} R K\right) e^{A_{c}(\theta, K) t} d t x_{0} \\
& =x_{0}^{T} P x_{0} .
\end{aligned}
$$

We further know that $P=P^{T}$ is the unique positive definite solution of the Lyapunov equation

$$
0=A_{c}(\theta, K)^{T} P+P A_{c}(\theta, K)+Q+K^{T} R K
$$

Hence, the above mentioned co-design problem can be formulated as the following optimization problem:

Problem 1 (Linear co-design problem):

$$
\begin{aligned}
\min _{\theta, K, P} & x_{0}^{T} P x_{0} \\
\text { s.t. } & \text { 1) } 0=A_{c}(\theta, K)^{T} P+P A_{c}(\theta, K)+Q+K^{T} R K \\
& \text { 2) } A_{c}(\theta, K) \text { is Hurwitz } \\
& \text { 3) } \theta_{\min } \leq \theta \leq \theta_{\max } .
\end{aligned}
$$

Remark 1: Solving this problem is challenging for at least two reasons. First, due to the coupling between the system parameters and the control input, Constraint (7) is non-convex with respect to $\theta, K$, and $P$ as decision variables. Second, Constraint (8) is not only nonlinear but also difficult to be formulated analytically, especially for high-dimensional systems. Also, as shown in Toker and Özbay (1995), finding $\theta$ to ensure the stability of $A_{c}$, equivalently the salification of (8), is an $N P$-hard problem.

Remark 2: For a general cost function

$$
J\left(x_{0}, \theta, K\right)=\int_{0}^{\infty}\left(x^{T} Q x+2 x^{T} S u+u^{T} R u\right) d t, x(0)=x_{0}
$$

where $S \in \mathrm{R}^{n \times m}$, the corresponding linear co-design problem takes a similar form as Problem 1 . The only difference is that (7) is replaced with the following equality

$$
A_{c}(\theta, K)^{T} P+P A_{c}(\theta, K)+Q+\left(S K+K^{T} S^{T}\right)+K^{T} R K=0
$$

where $P$ is the finite cost matrix associated with the stabilizing control policy $u=K x$. The additional term $S K+K^{T} S^{T}$, which is linear in the decision variable $K$, does not introduce any difficulty in solving Problem 1, and thus this paper only considers Problem 1 to simplify the presentation. 
Different from the conventional system design process, where the design is to seek a feasible solution, the co-design process seeks an optimal solution which consequentially gives rise to an optimization problem. The resultant linear co-design problem formulation is exposed to several fundamental questions: does the problem have an optimal solution, is it unique, and how to compute it. Limited work, e.g. Cesari (1965); Filippov (1962), has been done on the existence conditions of an optimal solution for a certain class of optimization problems partially due to its difficulty, even if the importance is widely acknowledged. Instead, many literatures assume the existence of optimal solutions and investigate the mathematical characterizations of optimal solutions, e.g. necessary optimality conditions for state constrained optimal control problems (Jacobson et al., 1971), maximum principle for hybrid systems (Sussmann, 1999), KKT conditions for nonlinear programming (Harold and Tucker, 1951), sufficiency of Pontryagin's necessary conditions (Mangasarian, 1966).

Similarly, there is limited work on the existence of optimal solutions for optimization problems derived from the co-design process. Papalambros and Wilde (2000) study the existence of an optimal solution of a system design problem using the Weierstrass Theorem, which requires the compactness of the feasible set. Some researchers have been endeavoring in developing necessary conditions for local optimal solutions (Alyaqout et al., 2007; Fathy et al., 2001; Patil et al., 2012; Peters et al., 2010, 2011). In terms of how to compute an optimal solution, one of the earliest studies of Problem 1 can be found in (Salama et al., 1988), where a gradient method was developed to numerically search for the optimal solution. The stability and convergence analysis of this method is however difficult to perform. Problem 1 has also been revisited in (Grigoriadis et al., 1993; Lu and Skelton, 2000; Skelton and Kim, 1992), where disturbances are taken into account, and iterative schemes based on the system equivalence constraint are proposed. All of the abovementioned iterative methods try to compute sub-optimal solutions of Problem 1, and do not guarantee to find a local optimum. This is because the computed sub-optimal solutions only satisfy necessary conditions, which are not sufficient to ensure the optimality. Also, due to its non-convexity property, the optimization problem may have multiple optimal solutions.

This paper assumes the existence of optimal solutions and focuses on the development of a novel and more general iterative method that computes a sub-optimal solution to Problem 1 without imposing the conservative constraint introduced in (Grigoriadis et al., 1993; Lu and Skelton, 2000; Skelton and Kim, 1992). In other words, we aim to develop algorithms which provide a good tradeoff among convergence, reliability, optimality, and computation burden. Note that iterative techniques based on the system equivalence constraint can be employed to solve this problem. The system-equivalence-based method, when applied to Problem 1, is summarized as follows for completeness.

Given $\theta_{0}$ satisfying $\theta_{\min } \leq \theta_{0} \leq \theta_{\max }$, repeat the following two steps with $i=0,1, \cdots$, until convergence. 1) Linear-quadratic-regulator $(L Q R)$ design

Solve for $P_{i}=P_{i}^{T}>0$ from

$$
0=A_{c}\left(\theta_{i}, K_{i}\right)^{T} P_{i}+P_{i} A_{c}\left(\theta_{i}, K_{i}\right)+Q+K_{i} R K_{i}
$$

where $K_{i}=-R^{-1} B^{T} P_{i}$.

2) System-equivalence-based redesign

Improve the system parameters and the control policy together by solving the optimization problem.

$$
\begin{aligned}
& \left.\qquad \theta_{i+1}, \bar{K}_{i}\right)=\underset{\theta, K}{\arg \min } J\left(x_{0}, \theta, K\right) \\
& \text { s.t } 1) A_{c}(\theta, K)=A_{c}\left(\theta_{i}, K_{i}\right) \\
& \text { 2) } \theta_{\min } \leq \theta \leq \theta_{\max }
\end{aligned}
$$

Remark 3: The feasible set of the optimization problem (11)-(13) is more restrictive compared with Problem 1, due to the conservative constraint (12). Also, solving the problem (11)-(13) is non-trivial because the constraint (12) is generally non-convex. 


\section{A novel iterative technique for solving the co-design problem}

In this section, we propose a novel iterative method to solve Problem 1. That is, the design of $K$ and $\theta$ is separated from each other. Given $\theta$ fixed, the feedback gain matrix $K$ can be readily determined using linear optimal control theory (Lewis et al., 2012). It is of particular interest to develop a general method for obtaining the optimal $\theta$, given fixed $K$. Our main contribution is to come up with a novel iterative method which provides less conservative designs of plant parameters in each iteration.

\subsection{Optimal system design}

Given a pair $\left(\theta_{i}, K_{i}\right)$ with $\theta_{\min } \leq \theta_{i} \leq \theta_{\max }$, and $A_{c}\left(\theta_{i}, K_{i}\right)$ Hurwitz, we give the following optimal system design problem.

Problem 2 (Optimal system design problem):

$$
\begin{array}{ll} 
& \left(\theta_{i+1}, S_{i}\right)=\arg \min _{\theta, S} x_{0}^{T} S x_{0} \\
\text { s.t. } 1) A_{c}\left(\theta, K_{i}\right)^{T} S+S A_{c}\left(\theta, K_{i}\right)+Q+K_{i}^{T} R K_{i}=0 \\
\text { 2) } A_{c}\left(\theta, K_{i}\right) \text { is Hurwitz } \\
\text { 3) } \theta_{\min } \leq \theta \leq \theta_{\max } .
\end{array}
$$

Note that (10) is the Riccati equation, and $P_{i}$ denotes the optimal cost matrix for a given $\theta_{i}$. On the other hand, given $\left(\theta, K_{i}\right), S$ in Problem 2 denotes the cost matrix satisfying the Lyapunov equation (15). While presenting the main idea, we use various notations of cost matrices, e.g. $P$ in Problem 1, $S$ in Problem 2, and $\bar{S}$ in Problem 3 later, to emphasize that decision variables in these three problems are different.

Problem 2 is non-convex due to constraints (15)-(16). The main novelty of the proposed approach lies in the treatment of the non-convex constraints (15)-(16). As a first step, we relax Problem 2 by replacing the first constraint (15) with an inequality constraint. The relaxation technique is standard and not surprising. We however can show that both Problem 2 and the relaxed problem have the same optimal solutions. Finally, we establish a set of simplified sufficient conditions which not only imply the non-convex constraints (15)-(16), but also admit less computation load. The relaxed problem is given below.

Problem 3 (Relaxed optimal system design problem):

$$
\begin{array}{ll} 
& \left(\bar{\theta}_{i+1}, \bar{S}_{i}\right)=\arg \min _{\bar{\theta}, \bar{S}} x_{0}^{T} \bar{S} x_{0} \\
\text { s.t. } & \text { 1) } A_{c}\left(\bar{\theta}, K_{i}\right)^{T} \bar{S}+\bar{S} A_{c}\left(\bar{\theta}, K_{i}\right)+Q+K_{i}^{T} R K_{i} \leq 0 \\
& \text { 2) } A_{c}\left(\bar{\theta}, K_{i}\right) \text { is Hurwitz } \\
& \text { 3) } \theta_{\min } \leq \bar{\theta} \leq \theta_{\max } .
\end{array}
$$

Problem 3 has a linear cost function and its constraints can be reformulated as bilinear matrix inequalities. Thus Problem 3 can be approached by existing BMI algorithms, e.g. global methods Beran et al. (1997); Goh et al. (1994); Tuan and Apkarian (2000); Tuan et al. (1999); Visweswaran et al. (1996), and local methods Hassibi et al. (1999); Iwasaki (1999); Kanev et al. (2004). Global methods typically work on the Lagrangian dual problem and use the branch-and-bound technique, and cannot yield solutions within polynomial-time. Local methods enjoy low computation burden, though the convergence to local optimal solutions is usually not guaranteed.

Lemma 1: The optimal cost of Problem 3 and the optimal cost of Problem 2 are the same, i.e., $x_{0}^{T} S_{i} x_{0}=$ $x_{0}^{T} \bar{S}_{i} x_{0}$. 
Proof: First, one can see that the optimal solution $\left(\theta_{i+1}, S_{i}\right)$ of Problem 2 is a feasible solution of Problem 3. Since the optimal solution of a minimization problem always gives less value of the object function that any feasible solution does, we have

$$
x_{0}^{T} \bar{S}_{i} x_{0} \leq x_{0}^{T} S_{i} x_{0}
$$

where $x_{0}^{T} \bar{S}_{i} x_{0}$ and $x_{0}^{T} S_{i} x_{0}$ represent the minimums of Problems 3 and 2, respectively.

On the other hand, by definition, we have

$$
\begin{aligned}
x_{0}^{T} \bar{S}_{i} x_{0} & \geq x_{0}^{T} \int_{0}^{\infty} e^{A_{c}\left(\bar{\theta}_{i+1}, K_{i}\right)^{T} t}\left(Q+K_{i}^{T} R K_{i}\right) e^{A_{c}\left(\bar{\theta}_{i+1}, K_{i}\right) t} d t x_{0} \\
& \geq x_{0}^{T} \int_{0}^{\infty} e^{A_{c}\left(\theta_{i+1}, K_{i}\right)^{T} t}\left(Q+K_{i}^{T} R K_{i}\right) e^{A_{c}\left(\theta_{i+1}, K_{i}\right) t} d t x_{0} \\
& \geq x_{0}^{T} S_{i} x_{0}
\end{aligned}
$$

As a result, $x_{0}^{T} \bar{S}_{i} x_{0}=x_{0}^{T} S_{i} x_{0}$. The proof is thus complete.

Remark 4: Constraint (19) cannot be converted to linear constraints using the change of variables method widely adopted to solve linear matrix inequality (LMI) problems (Boyd et al., 1994). Let us assume $\bar{\theta} \in \mathrm{R}$, and $A_{c}\left(\bar{\theta}, K_{i}\right)=\bar{\theta} A_{c}$. Then, it is easy to see constraint (19) is equivalent to

$$
T A_{c}^{T}+A_{c} T+\bar{S}^{-1}\left(Q+K_{i}^{T} R K_{i}\right) \bar{S}^{-1} \leq 0
$$

where $T=T^{T}=\bar{\theta} \bar{S}^{-1}$, and (23) can be converted to an LMI if $T$ and $\bar{S}^{-1}$ are viewed as two independent matrices of decision variables and $Q+K_{i}^{T} R K_{i}>0$. However, once $S^{-1}$ and $T$ are determined, we need to recover $\bar{\theta}$ from

$$
\bar{\theta} I_{n}=T S
$$

which is a set of over-determined linear equations. To guarantee there is a unique solution of $\bar{\theta}$, we need to not only keep $\bar{\theta}$ as a decision variable but also impose the additional non-convex constraint (24).

Fortunately, due to the relaxation of the equality constraint, we are able to get around the non-convexity and nonlinearity in (15) and (16) by deriving some sufficient conditions for these two constraints using Schur complement condition (Boyd and Vandenberghe, 2004).

Let us define $P_{i}=P_{i}^{T}>0$ as the positive definite solution of

$$
A_{i}^{T} P_{i}+P_{i} A_{i}+Q+K_{i}^{T} R K_{i}=0 .
$$

where $A_{i}=A_{c}\left(\theta_{i}, K_{i}\right)$. Also, let $\Delta A_{i}(\bar{\theta})=A_{c}\left(\bar{\theta}, K_{i}\right)-A_{c}\left(\theta_{i}, K_{i}\right), \Delta P_{i}(\bar{S})=\bar{S}-P_{i}$, and $\bar{Q}$ denotes a symmetric matrix satisfying

$$
\begin{aligned}
0= & \left(P_{i}+\Delta P_{i}\right) A_{c}\left(\theta_{i+1}, K_{i}\right)+A_{c}\left(\theta_{i+1}, K_{i}\right)^{T}\left(P_{i}+\Delta P_{i}\right) \\
& +\bar{Q}+K_{i}^{T} R K_{i} .
\end{aligned}
$$

Then, the following lemma gives sufficient conditions for (19) and (20). 
Lemma 2: The constraints (19) and (20) are satisfied if the following two linear matrix inequalities hold.

$$
\begin{aligned}
& {\left[\begin{array}{ccc}
M(\bar{S}, \bar{\theta}) & \Delta P_{i}(\bar{S}) & \Delta A_{i}(\bar{\theta})^{T} \\
\Delta P_{i}(\bar{S}) & I_{n} & 0 \\
\Delta A_{i}(\bar{\theta}) & 0 & I_{n}
\end{array}\right] \geq 0} \\
& \bar{S}>0 .
\end{aligned}
$$

where

$$
\begin{aligned}
M(\bar{S}, \bar{\theta})= & -\Delta P_{i}(\bar{S}) A_{i}-A_{i}^{T} \Delta P_{i}(\bar{S}) \\
& -\Delta A_{i}(\bar{\theta})^{T} P_{i}-P_{i} \Delta A_{i}(\bar{\theta})
\end{aligned}
$$

Proof: For simplicity and without causing confusion, in this proof we drop the argument $(\bar{S})$ from $\Delta P_{i}$, and the argument $(\bar{\theta})$ from $\Delta A_{i}$. By Schur complement condition, inequality (27) holds if and only if

$$
-\Delta P_{i} A_{i}-A_{i}^{T} \Delta P_{i}-\Delta A_{i}^{T} P_{i}-P_{i} \Delta A_{i}-\left(\Delta P_{i}\right)^{2}-\Delta A_{i}^{T} \Delta A_{i} \geq 0
$$

Therefore, by (19), (25), and (30) we have

$$
\begin{aligned}
& A_{c}\left(\bar{\theta}, K_{i}\right)^{T} \bar{S}+\bar{S} A_{c}\left(\bar{\theta}, K_{i}\right)+Q+K_{i}^{T} R K_{i} \\
= & \left(A_{i}+\Delta A_{i}\right)^{T}\left(\Delta P_{i}+P_{i}\right)+\left(\Delta P_{i}+P_{i}\right)\left(A_{i}+\Delta A_{i}\right)+Q+K_{i}^{T} R K_{i} \\
= & \left(A_{i}+\Delta A_{i}\right)^{T}\left(\Delta P_{i}+P_{i}\right)+\left(\Delta P_{i}+P_{i}\right)\left(A_{i}+\Delta A_{i}\right)-P_{i} A_{i}-A_{i}^{T} P_{i} \\
= & A_{i}^{T} \Delta P_{i}+\Delta P_{i} A_{i}+P_{i} \Delta A_{i}+\Delta A_{i}^{T} P_{i}+\Delta P_{i} \Delta A_{i}+\Delta A_{i}^{T} \Delta P_{i} \\
\leq & A_{i}^{T} \Delta P_{i}+\Delta P_{i} A_{i}+P_{i} \Delta A_{i}+\Delta A_{i}^{T} P_{i}+\left(\Delta P_{i}\right)^{2}+\Delta A_{i}^{T} \Delta A_{i} \\
\leq & 0 .
\end{aligned}
$$

From the fact that $Q$ and $\bar{Q}$ satisfy (31) and (26), respectively, it is not difficult to derive $\bar{Q} \geq Q$. Since $\left(A(\bar{\theta}), Q^{1 / 2}\right)$ is assumed to be observable and $\bar{Q} \geq Q$, the pair $\left(A(\bar{\theta}), \bar{Q}^{1 / 2}\right)$ is also observable from its observability Gramian. In addition, because $\bar{S}$ is positive definite, we know $A_{c}\left(\bar{\theta}, K_{i}\right)$ must be Hurwitz. The proof is complete.

Remark 5: Conditions (27) and (28) give two linear matrix inequalities when $\Delta P_{i}(\bar{S})$, equivalently $\bar{S}$, and $\Delta A_{i}(\bar{\theta})$ are decision variables. If we further assume the system matrices $A$ and $B$ have linear parametrization over $\theta$, i.e., $\Delta A_{i}(\bar{\theta})=A\left(\bar{\theta}-\theta_{i}\right)+B\left(\bar{\theta}-\theta_{i}\right) K_{i}$, (27) and (28) give two linear matrix inequalities in terms of decision variables $\bar{S}$ and $\bar{\theta}$. Replacing (19) and (20) with (27) and (28), Problem 3 turns into a semi-definite programming (SDP) problem (Boyd and Vandenberghe, 2004) which does not involve non-convexity or nonlinearity. The SDP problem can be solved within polynomial time using, for instance, interior point methods (Wolkowicz et al., 2000) and first order methods (Wen et al., 2010). The latter is commonly based on the augmented Lagrangian and has variants including alternating direction methods (He et al., 2011; Wen et al., 2010).

Remark 6: System parameter constraints (13) are not necessarily lower- and upper-bounded. Consider more general system parameter constraints: $\underline{h}(\theta) \leq \theta \leq \bar{h}(\theta)$, where $\underline{h}(\theta)=\left(\underline{h}_{1}(\theta), \ldots, \underline{h}_{n}(\theta)\right)^{T}$ and $\bar{h}(\theta)=\left(\bar{h}_{1}(\theta), \ldots, \bar{h}_{n}(\theta)\right)^{T}$. If $\underline{h}_{i}(\theta)$ and $\bar{h}_{i}(\theta)$ for $1 \leq i \leq n$ are linear in $\boldsymbol{\theta}$, the main results established in this paper are still valid, i.e., Problem 3 is reduced to an SDP problem. Furthermore, if $\underline{h}_{i}(\theta)$ and $\bar{h}_{i}(\theta)$ for $1 \leq i \leq n$ are convex in $\theta$, Problem 3 is reduced to a convex programming problem. 


\subsection{An iterative technique to solve the co-design problem}

Now we are ready to develop an iterative technique for solving Problem 1.

1) Initialization

Let $\theta_{0}$ and $K_{0}$ satisfy $\theta_{\min } \leq \theta_{0} \leq \theta_{\max }$, and $A_{c}\left(\theta_{0}, K_{0}\right)$ is Hurwitz. Let $i=0$.

2) Policy evaluation

Solve for $P_{i}=P_{i}^{T}>0$ from

$$
0=A_{i}^{T} P_{i}+P_{i} A_{i}+Q+K_{i}^{T} R K_{i}
$$

where $A_{i}=A_{c}\left(\theta_{i}, K_{i}\right)$.

3) Optimal system design

$$
\begin{array}{ll} 
& \left(\theta_{i+1}, \Delta P_{i}\right)=\arg \min _{\theta, \Delta P} x_{0}^{T} \Delta P x_{0} \\
\text { s.t. } & {\left[\begin{array}{ccc}
-\Delta P A_{i}-A_{i}^{T} \Delta P-\Delta A_{i}^{T} P_{i}-P_{i} \Delta A_{i} \Delta P & \Delta A_{i}^{T} \\
\Delta P & I_{n} & 0 \\
\Delta A_{i} & 0 & I_{n}
\end{array}\right] \geq 0} \\
& \Delta P+P_{i}>0 \\
& \theta_{\min } \leq \theta \leq \theta_{\max }
\end{array}
$$

where $\Delta A_{i}=A\left(\theta-\theta_{i}\right)+B\left(\theta-\theta_{i}\right) K_{i}$.

4) Policy improvement

Update the control policy by

$$
K_{i+1}=-R^{-1} B\left(\theta_{i+1}\right)^{T}\left(P_{i}+\Delta P_{i}\right)
$$

\section{5) Stopping criterion}

Stop, if $\left\|P_{i}-P_{i-1}\right\|<\varepsilon$, with $\varepsilon>0$ a predefined sufficiently small constant. Otherwise, go to Step 2) with $i$ replaced by $i+1$.

Remark 7: In the absence of Step 3), (i.e., when $\theta$ is fixed) the algorithm described in (32)-(37) is reduced to the well-known Kleinman algorithm (Kleinman, 1968) for solving algebraic Riccati equations.

Remark 8: Compared to the system-equivalence-based methods where the system design problem is likely non-convex, the proposed approach resorts to convex optimization problems which can always be solved in polynomial time, thus generally requires less computation load.

Theorem 1: For $i=0,1,2, \cdots$, the following properties of the algorithm described in (32)-(36) hold.

1) $A_{i}$ is Hurwitz.

2) $0 \leq x_{0}^{T} P_{i+1} x_{0} \leq x_{0}^{T}\left(P_{i}+\Delta P_{i}\right) x_{0} \leq x_{0}^{T} P_{i} x_{0}$.

3) There exists $J^{*}>0$, such that $\lim _{i \rightarrow \infty} J\left(x_{0}, \theta_{i}, K_{i}\right)=J^{*}$.

Proof: 1) We prove by induction. i) First, by the assumption in the algorithm we know $A_{0}$ is Hurwitz. ii) Suppose $A_{i}$ is Huwritz, let us show $A_{i+1}$ is also Hurwitz. Indeed, since $P_{i}+\Delta P_{i}>0$, by Lemma 2 we know $A_{c}\left(\theta_{i+1}, K_{i}\right)$ is Hurwitz.

Then, by (34) and Lemma 2, we know $\bar{Q} \geq Q$. Notice that (26) and (37) can be viewed as a single iteration step described in the Kleinman algorithm (Kleinman, 1968). Hence, by the main theorem in Kleinman (1968), $A_{i+1}$ is Hurwitz. 
2) By 1), $A_{i+1}$ is Hurwitz. Also, since $\left(A\left(\theta_{i+1}\right), Q^{1 / 2}\right)$ is assumed to be observable, $P_{i+1}$ is a symmetric positive definite matrix. Therefore, the first inequality holds.

Further, by Lemma 1 and the property of the Kleinman algorithm (Kleinman, 1968), we have

$$
\begin{aligned}
& \left(P_{i}+\Delta P_{i}\right)-P_{i+1} \\
\geq & \int_{0}^{\infty} e^{A_{i+1}^{T} t}\left(K_{i}-K_{i+1}\right)^{T} R\left(K_{i}-K_{i+1}\right) e^{A_{i+1} t} d t \geq 0 .
\end{aligned}
$$

Hence, the second inequality in 2) is proved.

To prove the last inequality in 2), we only need to show

$$
x_{0}^{T} \Delta P_{i} x_{0} \leq 0
$$

Notice that $\Delta P=0$ and $\theta=\theta_{i}$ is a feasible solution of the optimization problem (33)-(36), with the cost equal to zero. As a result, (39) holds.

3) The sequence $\left\{J\left(x_{0}, \theta_{i}, K_{i}\right)\right\}_{i=0}^{\infty}$ is non-negative and monotonically decreasing. Therefore, the limit of the sequence exists.

\subsection{Discussions}

Results obtained from the proposed method depend on the initial condition of the system state. This dependency makes sense in a class of practical engineering problems. For example, the general task of the load positioning problem described in the next section is to drive the load from the equilibrium to track a step response. As a result, its initial condition in the space is $x(0)=\lambda x_{0}$ with $x_{0}=[-1,0,0,0]^{T}$ and $\lambda$ a constant. As a result, there is no need to recalculate the control policy and the system parameters with different $\lambda$, because solving Problem 1 is equivalent to solving the same problem with (6) replaced by $\min _{\theta, K}=\lambda^{2} x_{0}^{T} P x_{0}$.

To reduce the sensitivity with respect to the error between the actual initial state and its nominal value, the following schemes can be adopted:

1) Consider the weighted sum of costs with different initial conditions

$$
\min _{\theta, K} \sum_{i=1}^{N} w_{i} J\left(x_{i}, \theta, K\right)
$$

where $x_{1}, \cdots, x_{N}$ are $N$ possible initial state with $w_{1}, \cdots, w_{N}>0$ being their weights, which are inspired by the state-relevance weights (de Farias and Van Roy, 2003). Notice that this modification will not affect the linearity in the optimal system design problem (33)-(36).

2) Replace the constraint (35) with

$$
-P_{i}<\Delta P \leq 0
$$

It implies that, the cost will be less or equal to the LQR cost with $\theta=\theta_{0}$ for all initial conditions. Again, solvability of the problem will not be affected.

It is also interesting to completely remove the dependency on the initial condition by considering the worse-case scenario for all possible initial conditions. Indeed, we can study the following cost

$$
\min _{\theta, K} \max _{\left|x_{0}\right|^{2}=1} J\left(x_{0}, \theta, K\right)
$$

This can be achieved by reformulating Problem 1 as an eigenvalue problem (EVP) (Boyd et al., 1994). 
Figure 1. Lumped parameter model of a motor-driven linear ballscrew stage supported on a flexible base platform bolted to the ground.

Problem 4 (Worst-case scenario co-design problem):

$$
\begin{aligned}
\min _{\theta, K, P, \mu} & \mu \\
\text { s.t. } & \\
& \text { 2) } A_{c}(\theta, K) \text { is Hurwitz } \\
& \text { 3) } \theta_{\min } \leq \theta \leq \theta_{\max } . \\
& \text { 4) } \mu I_{n}-P \geq 0 .
\end{aligned}
$$

This problem can be solved by a trivial extension of the proposed iterative method. Furthermore, the initial-condition-dependency can also be removed by considering the $H_{2}$ and $H_{\infty}$ gains for the closed-loop system with respect to external disturbance input (Lu and Skelton, 2000).

Finally, it is worth noticing how the feasible set of Problem 3 is restricted when the first two constraints (19) and (20) are replaced with (27) and (28). To study this, first notice that, in (31), we utilized the following inequathts

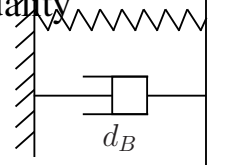

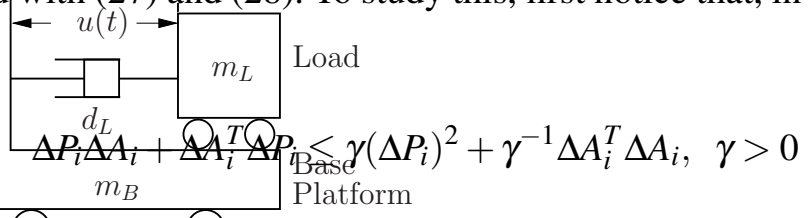

and condition (27) is deryed $x$ urth 1 th $x$ It is the only part in which the feasible set of Problem 3 is restricted. Therefore, it will be interesting to replace (34) with

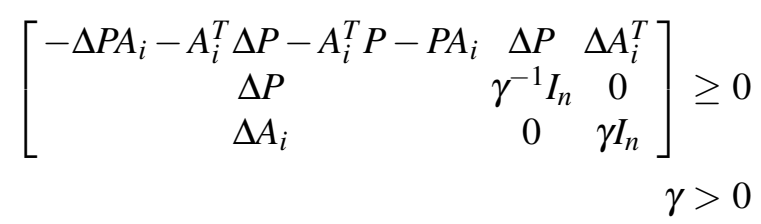

and to see if better solutions can be obtained with $\gamma \neq 1$. However, with $\gamma$ involved, the constraints are nonlinear and solving the optimization problem comprised of (33), (36), (49), and (50) becomes non-trivial.

\section{Application to a load-positioning problem}

In this section, we applied the proposed optimal co-design method to a mechanical system as shown in Figure 1. Its dynamics can be described by the following continuous-time linear time-invariant system 
(Shilpiekandula et al., 2012):

$$
\begin{aligned}
& \ddot{x}_{L}=\left(u-d_{L} \dot{x}_{L}\right)\left(\frac{1}{m_{L}}+\frac{1}{m_{B}}\right)+\frac{k_{B}}{m_{B}} x_{B}+\frac{d_{B}}{m_{B}} \dot{x}_{B} \\
& \ddot{x}_{B}=\left(d_{L} \dot{x}_{L}-u\right) \frac{1}{m_{B}}-\frac{k_{B}}{m_{B}} x_{B}-\frac{d_{B}}{m_{B}} \dot{x}_{B}
\end{aligned}
$$

where $x_{L}$ and $x_{B}$ denote the relative displacement of the load with respect to the platform, and the displacement of the platform. $d_{L}, m_{B}, m_{L}, k_{B}$, and $d_{B}$ are system parameters to be designed.

The co-design objective is to optimize the system performance in tracking a step command. For this purpose, we define $x_{1}=x_{L}-y_{d}$, with $y_{d}$ the desired constant output, $x_{2}=\dot{x}_{L}, x_{3}=x_{B}$, and $x_{4}=\dot{x}_{B}$. The system is converted to

$$
\dot{x}=A x+B u
$$

where $x=\left[x_{1}, x_{2}, x_{3}, x_{4}\right]^{T}$, and

$$
A=\left[\begin{array}{cccc}
0 & 1 & 0 & 0 \\
0-\frac{d_{L}}{m_{L}}-\frac{d_{L}}{m_{B}} & \frac{k_{B}}{m_{B}} & \frac{d_{B}}{m_{B}} \\
0 & 0 & 0 & 1 \\
0 & \frac{d_{L}}{m_{B}} & -\frac{k_{B}}{m_{B}} & -\frac{d_{B}}{m_{B}}
\end{array}\right], \quad B=\left[\begin{array}{c}
0 \\
\frac{1}{m_{L}}+\frac{1}{m_{B}} \\
0 \\
-\frac{1}{m_{B}}
\end{array}\right]
$$

As shown in Remark 5, solving Problem 3 using linear matrix inequalities technique involves the linear parametrization of $\Delta A$ and $\Delta B$ or equivalently $A$ and $B$. It is evident that $A$ and $B$ are not linear over the original system parameters $\left[m_{B}, m_{L}, d_{L}, k_{B}, d_{B}\right]^{T}$, and thus re-parametrization is necessary to ensure the linear parameterizations condition. It is also not straightforward to linearly re-parameterize $A$ and $B$ without overparametrization. For simplicity, we fix $d_{L}=10$ to avoid over-parametrization. With $d_{L}$ fixed, the number of system parameters is reduced to 4 , and various re-parametrization schemes are viable. In the simulation, we consider new system parameters $\theta=\left[\frac{1}{m_{L}}, \frac{1}{m_{B}}, \frac{k_{B}}{m_{B}}, \frac{d_{B}}{m_{B}}\right]^{T}$, and compute their lower bounds, upper bounds, and initial values as shown in the second to fourth columns in Table 1. Given $u=K x$ and $\theta$, we have the linear parametrization: $\Delta A(\theta, K)=\Sigma_{i=1}^{4} A^{(j)} \delta \theta^{(i)}$ where $\delta \theta^{(j)}$ is the variation of $\theta^{(j)}$, and

$$
\begin{aligned}
A^{(1)} & =\left[\begin{array}{cccc}
0 & 0 & 0 & 0 \\
0 & -d_{L} & 0 & 0 \\
0 & 0 & 0 & 0 \\
0 & 0 & 0 & 0
\end{array}\right]+\left[\begin{array}{l}
0 \\
1 \\
0 \\
0
\end{array}\right] K, \\
A^{(2)} & =\left[\begin{array}{cccc}
0 & 0 & 0 & 0 \\
0 & -d_{L} & 0 & 0 \\
0 & 0 & 0 & 0 \\
0 & d_{L} & 0 & 0
\end{array}\right]+\left[\begin{array}{c}
0 \\
1 \\
0 \\
-1
\end{array}\right] K, \\
A^{(3)} & =\left[\begin{array}{llll}
0 & 0 & 0 & 0 \\
0 & 0 & 1 & 0 \\
0 & 0 & 0 & 0 \\
0 & 0 & -1 & 0
\end{array}\right], \quad A^{(4)}=\left[\begin{array}{llll}
0 & 0 & 0 & 0 \\
0 & 0 & 0 & 1 \\
0 & 0 & 0 & 0 \\
0 & 0 & 0 & -1
\end{array}\right] .
\end{aligned}
$$

Alternative definition of the system parameter after re-parametrization could be $\theta=\left[\frac{1}{m_{L}}+\frac{1}{m_{B}}, \frac{1}{m_{B}}, \frac{k_{B}}{m_{B}}, \frac{d_{B}}{m_{B}}\right]^{T}$. 
Convergence performance

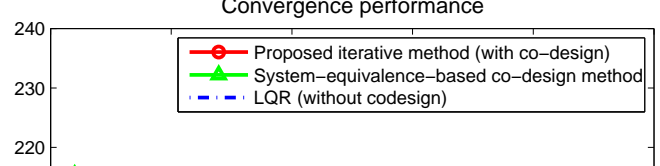

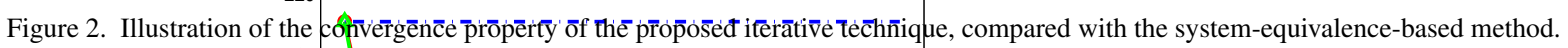
200

The cost to be minimized is ehosen as

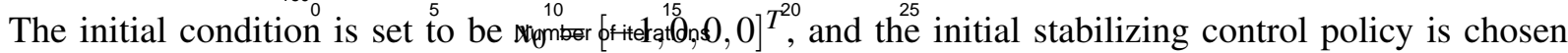
as the LQR control policy. The initial cost is $J=215.3952$. The stopping criterion is set to $\varepsilon=0.02$. The algorithm stops after 24 iterations. For comparison purpose, we have also computed this problem using the system-equivalence-based method described in (10)-(12). The optimized parameters are shown in the fifth column of Table 1. The results obtained using the system-equivalence-based method are provided in the last column of Table 1, and it can be seen that the ratios $\frac{m_{L}}{m_{B}}, \frac{k_{B}}{m_{B}}$, and $\frac{d_{B}}{m_{B}}$ are not changed at all, due the conservative constraint (12). Note that optimal system parameters computed by the proposed algorithm in this example tend to reach the bounds. This could be due to various factors such as the cost function. Intuitively, $\theta_{1}$ and $\theta_{2}$ should reach the upper bounds corresponding to the smallest system. Determination of other parameters $\theta_{3}$ and $\theta_{4}$ could be counter-intuitive. This is especially true for co-design of complex systems. In fact, if the weight on $u$ in the cost function is 1 , the proposed algorithm gives a design solution: $\theta^{*}=[1,0.0667,0.4,0.03]^{T}$, and the associated cost function value is less than the bound-based design solution: $\theta^{*}=[1,0.0667,0.4,0.004]^{T}$.

In Figure 2, we see the proposed algorithm yields a system with a cost $J=169.5836$, while the cost corresponding to the system-equivalence-based method is $J=193.1239$. Figures 3 and 4 also show that a shorter settling time can be achieved while less control energy is required, after applying the proposed co-design algorithm. Figure 3 however shows the load position trajectory tracking a unit step input from the zero initial state. This is based on the understanding that for a linear system (53), regulating the state from $x_{0}=[-1,0,0,0]^{T}$ to zero, implemented in simulation, is equivalent to controlling the zero-state system to track a unit step reference, as shown in Figure 3.

Table 1. System parameters

\begin{tabular}{|c|c|c|c|c|c|}
\hline Param. & Min. & Max. & Init. & Opt. & S.E.-based \\
\hline$\frac{1}{m_{L}}$ & 0.3333 & 1 & 0.5 & 1 & 0.6667 \\
\hline$\frac{1}{m_{B}}$ & 0.04 & 0.0667 & 0.05 & 0.0667 & 0.0667 \\
\hline$\frac{k_{B}}{m_{B}}$ & 0.4 & 1.3333 & 0.75 & 0.4393 & 0.75 \\
\hline$\frac{d_{B}}{m_{B}}$ & 0.004 & 0.0667 & 0.025 & 0.0369 & 0.025 \\
\hline
\end{tabular}




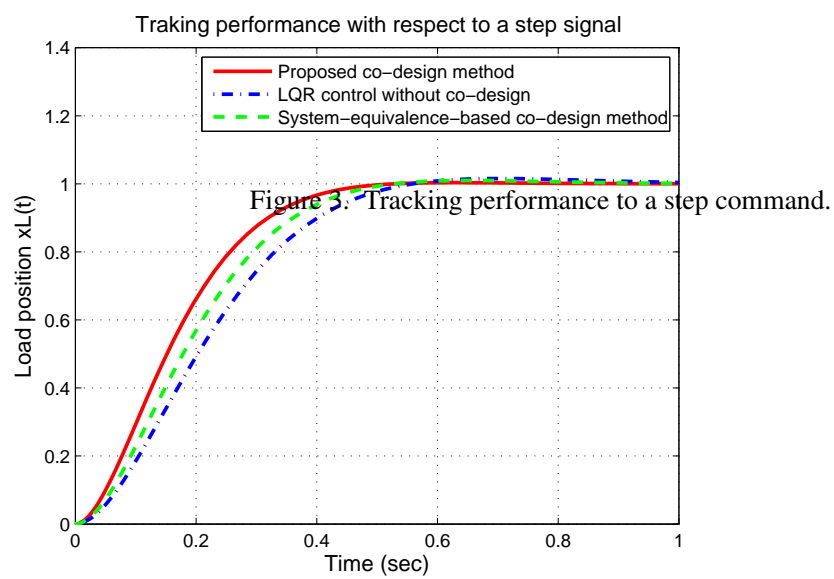

\section{Conclusions}

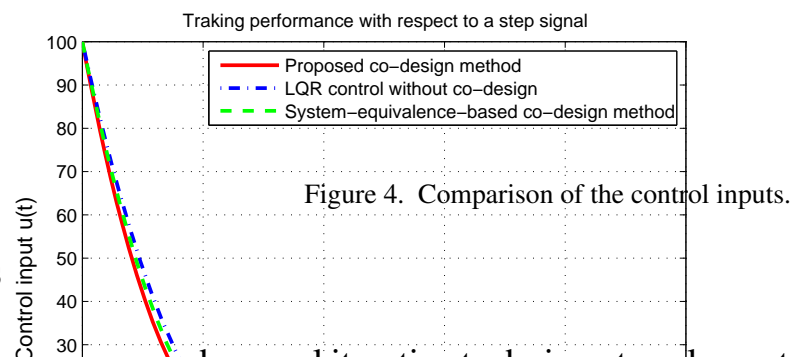

In this paper, we hăve proposed a novel iterative technique to solve optimal co-design problems for a class of linear control systems. After separating each iteration step into an optimal control problem and an optimal system design problem, we have shown that the second problem can be achieved by solving a standard semi-definite programming problem, wìthoü in involving non-convexity and nonlinearity. We have proposed

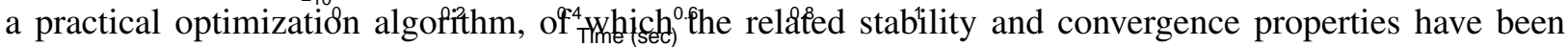
proved. The application to the co-design of a load positioning example has been investigated to validate the efficiency of the proposed algorithm. It will be interesting to extend the proposed method to study the co-design of nonlinear systems.

\section{References}

Alyaqout, S. F., Papalambros, P. Y., and Ulsoy, A. G. (2007). Coupling in design and robust control optimization. In Proceedings of the European Control Conference, Kos, Greece.

Beran, E., Vandenberghe, L., and Boyd, S. (1997). A global BMI algorithm based on the generalized benders decomposition. In Proceedings of the European Control Conference, pages 1074-1082, Brussels, Belgium.

Bertsekas, D. P. (1995). Dynamic Programming and Optimal Control. Athena Scientific Belmont.

Bode, H. W. (1945). Network Analysis and Feedback Amplifier Design. Van Nostrand.

Boyd, S. P., El Ghaoui, L., Feron, E., and Balakrishnan, V. (1994). Linear Matrix Inequalities in System and Control Theory, volume 15. SIAM, Philadelphia. 
Boyd, S. P. and Vandenberghe, L. (2004). Convex Optimization. Cambridge University Press.

Bryson, A. E. and Ho, Y.-C. (1975). Applied Optimal Control: Optimization, Estimation, and Control. Taylor \& Francis Group.

Cesari, L. (1965). Existence theorems for optimal solutions in Pontryagin and Lagrange problems. SIAM Journal on Control, 3(3):475-498.

da Silva, M. M., Brüls, O., Desmet, W., and Van Brussel, H. (2009). Integrated structure and control design for mechatronic systems with configuration-dependent dynamics. Mechatronics, 19(6):1016-1025.

de Farias, D. P. and Van Roy, B. (2003). The linear programming approach to approximate dynamic programming. Operations Research, 51(6):850-865.

Fathy, H. K., Reyer, J. A., Papalambros, P. Y., and Ulsoy, G. (2001). On the coupling between the plant and the controller optimization problems. In Proceedings of the American Control Conferences, pages 1864-1869, Arlington, VA.

Filippov, A. F. (1962). On certain questions in the theory of optimal control. Journal of The Society for Industrial and Applied Mathematics, Series A: Control, 1(1):76-84.

Freudenberg, J. S. and Looze, D. P. (1985). Right half plane poles and zeros and design tradeoffs in feedback systems. IEEE Transactions on Automatic Control, AC-30(6):555-565.

Goh, K. C., Safonov, M. G., and Papavassilopoulos, G. P. (1994). A global optimization approach for the BMI problem. In Proceedings of the IEEE Conference on Decision and Control, pages 2009-2014, Lake Buena Vista, FL.

Grigoriadis, K. M., Carpenter, M. J., Zhu, G., and Skelton, R. E. (1993). Optimal redesign of linear systems. In Proceedings of the American Control Conference, pages 2680-2684, San Francisco, CA.

Hale, A. L., Dahl, W., and Lisowski, J. (1985). Optimal simultaneous structural and control design of maneuvering flexible spacecraft. Journal of Guidance, Control, and Dynamics, 8(1):86-93.

Harold, H. W. and Tucker, A. W. (1951). Nonlinear programming. In Proceedings of the Second Berkeley Symposium on Mathematical Statistics and Probability, volume 5.

Hassibi, A., Jonathan, H., and Boyd, S. (1999). A path-following method for solving BMI problems in control. In Proceedings of the American Control Conference, pages 1385-1389, San Diego, CA.

He, B., Xu, M., and Yuan, X. (2011). Solving large-scale least squares seimdefinite programming by alternating direction methods. SIAM Journal of Matrix Analysis and Applications, 32(1):136-152.

Iwasaki, T. (1999). The dual iteration for fixed-order control. IEEE Transactions on Automatic Control, 44(4):783788.

Jacobson, D. H., Lele, M. M., and Speyer, J. L. (1971). New necessary conditions of optimality for control problems with state-variable inequality constraints. Journal of Mathematical Analysis and Applications, 35:255-284.

Jiang, Y., Wang, Y., Bortoff, S. A., and Jiang, Z.-P. (2015). Optimal co-design of nonlinear control systems based on a modified policy iteration method. IEEE Transactions on Neural Networks and Learning Systems, 26(2):409-414.

Kanev, S., Scherer, C., Verhaegen, M., and De Schutter, B. (2004). Robust output-feedback controller design via BMI optimization. Automatica, 40(7):1115-1127.

Kleinman, D. (1968). On an iterative technique for Riccati equation computations. IEEE Transactions on Automatic Control, 13(1):114-115.

Lewis, F. L., Vrabie, D., and Syrmos, V. L. (2012). Optimal Control, 3rd Edition. Wiley.

Li, Q., Zhang, W. J., and Chen, L. (2001). Design for control-a concurrent engineering approach for mechatronic systems design. IEEE/ASME Transactions on Mechatronics, 6(2):161-169.

Lu, J. and Skelton, R. E. (2000). Integrating structure and control design to achieve mixed $H_{2} / H_{\infty}$ performance. International Journal of Control, 73(16):1449-1462.

Mangasarian, O. L. (1966). Sufficient conditions for the optimal control of nonlinear systems. SIAM Journal on Control, 4(1):139-152.

Messac, A. (1998). Control-structure integrated design with closed-form design metrics using physical programming. AIAA Journal, 36(5):855-864.

Onoda, J. and Haftka, R. T. (1987). An approach to structure/control simultaneous optimization for large flexible spacecraft. AIAA Journal, 25(8):1133-1138.

Papalambros, P. Y. and Wilde, D. J. (2000). Principles of Optimal Design. Cambridge University Press, UK.

Patil, R., Filipi, Z., and Fathy, H. (2010). Computationally efficient combined design and control optimization using a coupling measure. In Proceedings of the 5th IFAC Symposium on Mechatronic Systems, pages 144-151.

Patil, R., Filipi, Z., and Fathy, H. (2012). Computationally efficient combined design and control optimization using a coupling measure. ASME Journal of Mechanical Design, 134(7):071008.

Peters, D. L., Papalambros, P. Y., and Ulsoy, A. G. (2010). Relationship between coupling and the controllability 
grammian in co-design problems. In Proceedings of the American Control Conference, pages 623-628.

Peters, D. L., Papalambros, P. Y., and Ulsoy, A. G. (2011). Control proxy functions for sequential design and control optimization. Journal of Mechanical Design, 133:091007.

Pil, A. C. and Asada, H. H. (1996). Integrated structure/control design of mechatronic systems using a recursive experimental optimization method. IEEE/ASME Transactions on Mechatronics, 1(3):191-203.

Ravichandran, T., Wang, D., and Heppler, G. (2006). Simultaneous plant-controller design optimization of a two-link planar manipulator. Mechatronics, 16(3):233-242.

Reyer, J. A. and Papalambros, P. Y. (2002). Combined optimal design and control with application to an electric DC motor. Journal of Mechanical Design, 124(2):183-191.

Salama, M., Garba, J., Demsetz, L., and Udwadia, F. (1988). Simultaneous optimization of controlled structures. Computational Mechanics, 3(4):275-282.

Serön, M. M., Braslavsky, J. H., and Goodwin, G. C. (1997). Fundamental Limitations in Filtering and Control. Springer, Berlin.

Shilpiekandula, V., Bortoff, S. A., Barnwell, J. C., and El-Rifai, K. (2012). Load positioning in the presence of base vibrations. In Proceedings of the American Control Conference, pages 6282-6287, Montreal, Canada.

Skelton, R. E. and Kim, J. H. (1992). The optimal mix of structure redesign and active dynamic controllers. In Proceedings of the American Control Conference, pages 2775-2779, Chicago, IL.

Sussmann, H. J. (1999). A maximum principle for hybrid optimal control problems. In Proceedings of the IEEE Conference on Decision and Control, pages 425-430, Phoenix, AZ.

Toker, O. and Özbay, H. (1995). On the NP-hardness of solving bilinear matrix inequalities and simulataneous stabilization with static output feedback. In Proceedings of the American Control Conference, pages 2525-2526, Seattle, WA.

Tuan, H. D. and Apkarian, P. (2000). Low nonconvexity-rank bilinear matrix inequalities: algorithms and applications in robust controller and structure designs. IEEE Transactions on Automatic Control, 45(11):2111-2117.

Tuan, H. D., Apkarian, P., and Nakashima, Y. (1999). A new lagrangian dual global optimization algorithm for solving bilinear matrix inequalities. In Proceedings of the American Control Conference, pages 1851-1855, San Diego, CA.

Visweswaran, V., Floudas, C. A., Ierapetritou, M. G., and Pistikopoulos, E. N. (1996). A decomposition-based global optimization approach for solving bilevel linear and quadratic programs. In State of the Art in Global Optimization, pages 139-162. Springer.

Wen, Z., Goldfarb, D., and Yin, W. (2010). Alternating direction augmented Lagrangian methods for semidefinite programming. Mathematical Programming Computation, 2(3-4):203-230.

Wolkowicz, H., Saigal, R., and Vandenberghe, L., editors (2000). Handbook of Semidefinite Programming: Theory, Algorithms, and Applications, volume 27. Springer. 